\title{
CUMPLIMIENTO DE LA VACUNACIÓN CONTRA EL VIRUS DE LA HEPATITIS B EN RECIÉN NACIDOS DE LIMA Y CALLAO
}

\author{
Alfredo Borda-Olivas ${ }^{1, a^{*}}$, Ángel Florián Florián ${ }^{2, \mathrm{~b}^{*}}$, Edgar Montalvan Santillan ${ }^{1, \mathrm{c}^{*}}$, María Dedios Alegría ${ }^{1, \mathrm{~d}^{*}}$, \\ César Cabezas Sánchez ${ }^{1,3, d}$, Fernando Donaires Toscano ${ }^{1,3, d}$.
}

\begin{abstract}
RESUMEN
El objetivo del estudio fue determinar el cumplimiento de la vacunación contra el virus de la hepatitis B (VHB) en recién nacidos de Lima y Callao. Estudio de diseño descriptivo, transversal y multicéntrico, realizado en hospitales de alto nivel, públicos y privados. La información sobre la vacunación corresponde a recién nacidos durante una semana del 2015, y fue obtenida mediante consultas a los padres y revisión de reporte de los servicios de salud. El estudio se realizó en 12 establecimientos de salud incluyéndose a 935 madres y recién nacidos. La cobertura de vacunación dentro de las 24 horas de vida fue de $85,1 \%$ según el reporte de padres y $88,7 \%$ según el reporte de los servicios de salud. Se concluye que la cobertura la vacunación contra el VHB en recién nacidos dentro de las 24 horas de vida fue importante, pero aún alejada de metas ideales propuestas por el Ministerio de Salud y la Organización Mundial de la Salud.
\end{abstract}

Palabras claves: Hepatitis B; Vacunas contra Hepatitis B; Cobertura de Vacunación; Recién Nacido; Perú (fuente: DeCS BIREME).

\section{COMPLIANCE OF HEPATITIS-B VIRUS VACCINATION IN NEWBORNS IN LIMA AND CALLAO}

\begin{abstract}
The objective of the study was to determine compliance with hepatitis-B virus (HBV) in newborns in Lima and Callao A descriptive, cross-sectional, multicenter-design study was conducted in high level public and private hospitals. Information on vaccination corresponds to newborns during one week of 2015 and was obtained through consultations with parents and review of health service reports. The study was conducted in 12 health facilities including 935 mothers and newborns. Vaccination coverage within 24 hours of life was $85.1 \%$, according to the parents' report; and $88.7 \%$, according to the health services' report. It is concluded that the coverage of HBV vaccination in newborns within 24 hours of life was important, but still far from the ideal goals proposed by the Ministry of Health and the World Health Organization.
\end{abstract}

Keywords: Hepatitis B; Hepatitis B vaccines; Vaccination coverage; Newborn; Perú (source: MeSH NLM).

\section{INTRODUCCIÓN}

La Organización Mundial de la Salud (OMS) estima que existen cerca de 257 millones de personas que padecen infección crónica por el virus de la hepatitis $\mathrm{B}(\mathrm{VHB})$, y más de 887 mil personas mueren cada año como consecuencia de las complicaciones de hepatitis $B$, incluido cirrosis $y$ cáncer hepático ${ }^{(1,2)}$. A pesar de los avances en métodos diagnósticos y tratamientos, la infección por el VHB es considerada un problema de salud pública y en la mayoría de los casos es el resultado de una transmisión maternofetal durante el parto ${ }^{(1)}$.
Actualmente se dispone de una vacuna recombinante contra el VHB con una eficacia del 95\% para prevenir la infección y la aparición de una enfermedad crónica y cáncer de hígado por el VHB ${ }^{(1)}$. Esta vacuna se puede administrar desde el nacimiento y ha demostrado ser una estrategia segura y eficaz, no reportándose en niños, efectos adversos graves mientras se logra la seroprotección ${ }^{(3,4)}$.

En el Perú, la vacunación universal contra el VHB se inició el 2003, con la vacunación a los dos, cuatro y seis meses, además de la administración en recién nacidos (RN) ${ }^{(5)}$. La vacuna debe administrarse idealmente dentro de las 24

\footnotetext{
Centro Nacional de Salud Pública, Instituto Nacional de Salud. Lima, Perú.

Instituto Nacional de Salud del Niño. Lima, Perú.

Facultad de Medicina, Universidad Nacional Mayor de San Marcos. Lima, Perú

Médico pediatría, especialista en Medicina Preventiva y Salud Pública, magíster en Salud Pública; ${ }^{\text {b }}$ médico gastroenterólogo pediatra, magíster en Gerencia en Servicios de Salud; ${ }^{\mathrm{c}}$ médico cirujano, magister en Salud Pública; ${ }^{\mathrm{d}}$ médico infectólogo.

* El estudio se realizó cuando los autores fueron trabajadores de la institución señalada.

Recibido: 19/04/2018 Aprobado: 12/09/2018 En línea: 25/09/2018
}

Citar como: Borda-Olivas A, Florián A, Montalvan E, Dedios M, Cabezas C, Donaires F. Cumplimiento de la vacunación contra el virus de la hepatitis B en recién nacidos de hospitales de Lima y Callao. Rev Peru Med Exp Salud Publica. 2018;35(3):465-70. doi: 10.17843/rpmesp.2018.353.3619. 
horas de vida en RN con más de 2000 gramos de peso en madres con antígeno de superficie de la hepatitis $B$ (HBsAg) negativo; y dentro de las 12 horas cuando la madre es HBsAg positivo, además de la administración de la inmunoglobulina para el VHB (HBIG) $)^{(2,6,7)}$; recomendándose también en niños prematuros menores de 2000 gramos según los resultados de serología de la madre ${ }^{(8)}$.

A nivel mundial se han establecido metas de cobertura para la vacunación contra el VHB en RN dentro de las 24 horas. En el Perú, el Ministerio de Salud (MINSA) ha establecido en $79 \%$ el valor basal de referencia para la meta cobertura de vacunación y en $95 \%$ como meta de cobertura ideal anual para cada departamento ${ }^{(7)}$. Del mismo modo, la OMS ha establecido como meta para el 2020 y 2030 el $50 \%$ y $90 \%$, respectivamente ${ }^{(9)}$.

Los reportes en el país sobre la cobertura de vacunación contra el VHB en RN señalan en promedio una cobertura de $78 \%$ para el periodo $2010-2015^{(5)}$, obteniendo el departamento de Lima un $70,9 \%$ en el $2014{ }^{(10)}$. Dichos reportes son elaborados a partir de la información proveída por los servicios de salud, los cuales no siempre reflejan la cobertura real de la vacunación contra el VHB en RN, debido a que no siempre se tiene de forma clara el momento exacto de la administración de la vacuna ${ }^{(9)}$.

En ese sentido, el presente estudio tuvo como objetivo evaluar el cumplimiento de la vacunación contra la infección del VHB en RN dentro de las primeras 24 horas de vida en hospitales y clínicas de la ciudad de Lima y Callao.

\section{EL ESTUDIO}

Es un estudio de diseño descriptivo, transversal y multicéntrico. La población de estudio incluyó a los RN atendidos en hospitales públicos y clínicas privados de alto nivel (tercer nivel o superior) con funciones obstétricas y neonatales de Lima Metropolitana y Callao.

Los RN fueron identificados inicialmente en los libros de registro de los servicios de atención inmediata, siendo seguidos en los servicios de hospitalización, hasta el momento de su alta, por un máximo de 72 horas. Para mayor fiabilidad, el recojo de información de la vacunación fue obtenida de dos fuentes: a) Reporte de padres (RP), mediante una encuesta a la madre en los servicios de hospitalización (en caso de que no se encontraba la madre, el reporte se obtuvo del padre o tutor), y b) Reporte del servicio de salud (RSS), a través del personal de salud responsable del programa de inmunizaciones, mediante la revisión del registro de vacunación (electrónico o en papel), carnet de vacunación o historias clínicas, en ese orden de preferencia. Además, se recogió información de los datos epidemiológicos de la madre y $\mathrm{RN}$ de la historia clínica neonatal o historia clínica materna. Se incluyeron como variables de estudio al tipo de establecimiento de salud

\section{MENSAJES CLAVE}

Motivación para realizar el estudio. En general, al Perú se le ha ubicado entre los países de endemicidad intermedia para la infección por el virus de la hepatitis $\mathrm{B}(\mathrm{VHB})$, requiriéndose información sobre acciones realizada para la prevención de la transmisión vertical.

Principales hallazgos. Sólo en un tercio de los establecimientos estudiados se alcanzan la cobertura esperable de vacunación contra el VHB en recién nacidos dentro de las 24 horas de vida (95\%), siendo en promedio de $85,1 \%$ según el reporte de los padres y $88,7 \%$ según el reporte de los servicios de salud.

Implicancias. Es necesario reformar la prevención de la trasmisión materno perinatal del VHB, contando para ello con la participación del sector público y privado, con énfasis en la calidad de atención de salud.

(Hospitales/Institutos MINSA, EsSalud, Policía Nacional del Perú (PNP), clínicas privadas), edad materna (menos de 18 años, de 18 a 35, y mayor de 35 años), estado civil de la madre (casada, soltera, conviviente, viuda), nivel educativo materno (primaria incompleta/completa, secundaria incompleta/completa, superior incompleta/ completa), tipo de parto (vaginal, cesárea), sexo del RN, y peso del RN (menos de 2000 gramos, de 2000 a 2500, de 2500 a 4000, y mayor de 4000 gramos).

El recojo de información fue realizado durante una semana de forma continua (incluyendo sábados y domingos), en semanas que no incluían días feriados, ni coincidían con huelgas, aniversarios $\mathrm{u}$ otras actividades. El periodo de recojo de información se realizó entre abril a julio del 2015. Las personas que realizaron el recojo de información eran personal de salud ajeno al establecimiento de salud, siendo en número de uno para los establecimientos de salud con un máximo de 15 partos al día, dos para un máximo de 30 partos al día y tres para más partos.

Se realizó un análisis de las variables demográficas, utilizando para ello las medidas de resumen de variables cualitativas y de las variables cuantitativas con el programa estadístico Epi Info 7.

El estudio fue revisado y aprobado por el Comité de Ética del Instituto Nacional de Salud y los comités de ética de los establecimientos de salud; además, se solicitó el consentimiento informado de los participantes.

\section{HALLAZGOS}

El estudio se realizó en 12 establecimientos de salud de Lima Metropolitana (10) y el Callao (2), los cuales atienden un aproximado de $30 \%$ de nacimientos de su jurisdicción (11). 935 madres y RN participaron del estudio. La muestra de madres tuvo una media de edad de 27,7 años [desviación estándar (DE): 6,6; mediana: 27; rango: 14-49]. El 70,5\% tenían estado civil conviviente. El 50\% tenían educación secundaria completa y $21,3 \%$ superior completa. 
La muestra de RN tuvo una media de peso de nacimiento de 3348 gramos (DE: 523; rango: 1090-5400) (Tabla 1). De los 19 RN con peso menor de 2000 gramos, se identificaron cuatro vacunados, pero sin información de HBsAg positiva materna. La información de los RN menores de 2000 gramos no fue incluida en los análisis siguientes, debido a que no se contemplan la vacunación contra el VHB en el normativa nacional ${ }^{(7)}$.

El 80,8 \% (740/916) de casos contó con información sobre la hora de vacunación contra el VHB, el reporte provino del padre, servicio de salud o ambos.

En la Tabla 2 se muestra algunas características particulares de los establecimientos de salud. El Instituto Nacional

Tabla 1. Características de madres y recién nacidos de 12 establecimientos de salud de Lima y Callao, abril julio 2015.

\begin{tabular}{|c|c|c|}
\hline Característica & $\mathbf{n}$ & $\%$ \\
\hline \multicolumn{3}{|l|}{ Establecimiento de Salud* } \\
\hline Hospitales/Institutos MINSA ( $\mathrm{n}=7$ ) & 726 & 77,6 \\
\hline EsSalud ( $n=2)$ & 155 & 16,6 \\
\hline $\operatorname{PNP}(n=1)$ & 16 & 1,7 \\
\hline Clínicas privadas $(n=2)$ & 38 & 4,1 \\
\hline \multicolumn{3}{|l|}{ Edad materna (años) } \\
\hline$<18$ & 13 & 1,4 \\
\hline $18-35$ & 746 & 80,0 \\
\hline$>35$ & 173 & 18,6 \\
\hline \multicolumn{3}{|l|}{ Estado civil } \\
\hline Casada & 149 & 15,9 \\
\hline Soltera & 125 & 13,4 \\
\hline Conviviente & 659 & 70,5 \\
\hline Viuda & 2 & 0,2 \\
\hline \multicolumn{3}{|l|}{ Nivel educativo materno } \\
\hline Primaria incompleta & 5 & 0,5 \\
\hline Primaria completa & 15 & 1,6 \\
\hline Secundaria incompleta & 148 & 15,8 \\
\hline Secundaria completa & 467 & 49,9 \\
\hline Superior incompleta & 99 & 10,6 \\
\hline Superior completa & 199 & 21,3 \\
\hline \multicolumn{3}{|l|}{ Tipo de parto } \\
\hline Vaginal & 504 & 53,9 \\
\hline Cesárea & 431 & 46,1 \\
\hline \multicolumn{3}{|l|}{ Sexo del recién nacido } \\
\hline Mujer & 445 & 47,7 \\
\hline Varón & 488 & 52,3 \\
\hline \multicolumn{3}{|l|}{ Peso del recién nacido (gramos) } \\
\hline Menor de 2000 & 19 & 2,0 \\
\hline Entre 2000 y 2500 & 37 & 4,0 \\
\hline Entre 2500 y 4000 & 797 & 85,4 \\
\hline Mayor de 4000 & 80 & 8,6 \\
\hline
\end{tabular}

Materno Perinatal (INMP) es el establecimiento que aporta el mayor número de RN (27\%), una clínica particular no realizó ninguna vacunación, y en el Hospital Nacional Edgardo Rebagliati Martins el 91,8\% (89/97) de RN no tuvo reporte de hora de vacunación, sin embargo, un gran porcentaje de ellos fueron vacunados (RP=67\% y $\mathrm{RSS}=77 \%$ ). La proporción de $\mathrm{RN}$ vacunados cuyos padres no recibieron información sobre la vacunación fueron 6,8\% (62/916), siendo mayor en el Hospital Nacional Dos de Mayo (75,5\%).

Respecto a la hora de vacunación contra el VHB en RN se encontró una media de 14,9 horas de vida (DE: 8; mediana: 14,4; rango: 0 - 40,2) según el RP y una media de 13,1 horas de vida (DE: 8,8; mediana: 12,3; rango: 0 - 61,2) según el RSS.

La vacunación contra el VHB dentro de las 24 horas de vida fue de $85,1 \%$ según el RP y $88,7 \%$ según el RSS. La vacunación dentro de las 12 horas de vida fue $39,5 \%$ y $48,5 \%$, según el RP y RSS, respectivamente. La vacunación dentro de las dos horas de vida fue según el RP 4,7\% y según el RSS 11,4\% (Figura 1).

En la Figura 2 se muestra la cobertura de vacunación por establecimiento, observándose que sólo un 33\% (4/12) de ellos, alcanzaron coberturas ideales (95\%) de vacunación contra el VHB dentro de las 24 horas de vida. Se observa también una gran proporción de vacunación dentro de las 2 horas en el Hospital Nacional Dos de Mayo y Hospital San Bartolomé, los mismos que alcanzan el 100\% de vacunación contra el VHB dentro de las 24 horas de vida.

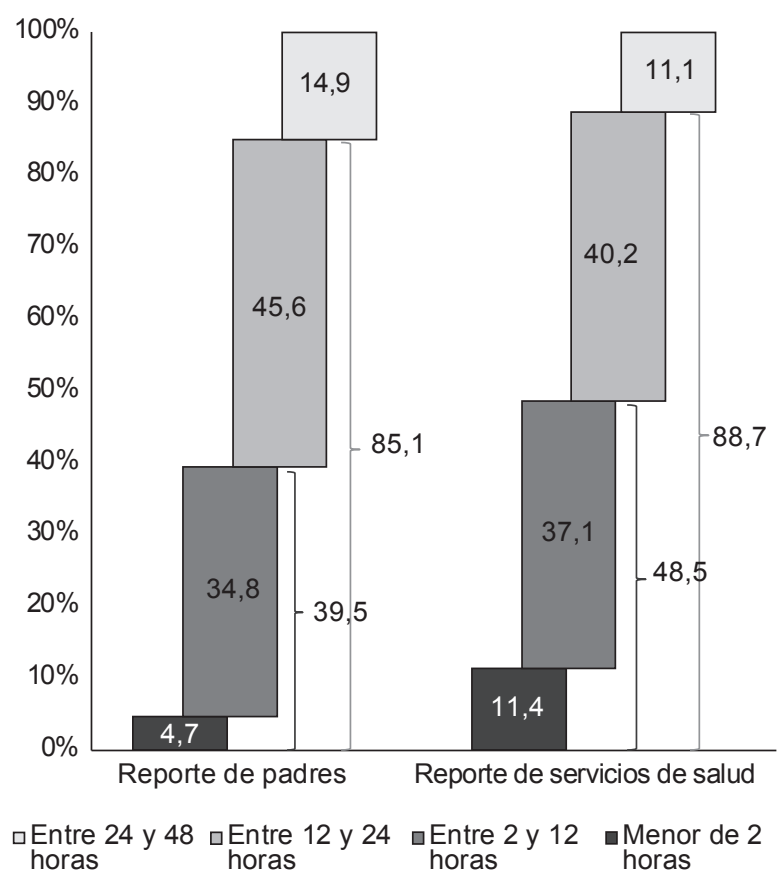

Figura 1. Cumplimiento de vacunación contra el VHB en RN según reporte de padres o servicios de salud y hora de vacunación $(n=740)$. 
Tabla 2. Condición de la vacunación y reporte de hora contra el VHB en recién nacidos en establecimientos de salud de Lima y Callao, abril - julio 2015

\begin{tabular}{|c|c|c|c|c|c|c|c|c|}
\hline \multirow{4}{*}{$\begin{array}{l}\text { Establecimiento de } \\
\text { salud }\end{array}$} & \multirow{4}{*}{$\begin{array}{c}\begin{array}{c}\text { Total de RN } \\
\text { del estudio }\end{array} \\
\mathbf{n}(\%)^{*}\end{array}$} & \multicolumn{3}{|c|}{ RN con reporte de hora de vacunación } & \multicolumn{3}{|c|}{$\begin{array}{c}\text { RN sin reporte de hora de } \\
\text { vacunación }\end{array}$} & \multirow{3}{*}{$\begin{array}{l}\text { RN vacunados } \\
\text { con padres no } \\
\text { informados de } \\
\text { la vacunación }\end{array}$} \\
\hline & & \multirow[b]{2}{*}{ Total } & \multirow[b]{2}{*}{ RP } & \multirow[b]{2}{*}{ RSS } & \multicolumn{3}{|c|}{ Vacunados } & \\
\hline & & & & & Total & RP & RSS & \\
\hline & & $\mathrm{n}(\%)$ & $\mathrm{n}(\%)$ & n (\%) & $\mathrm{n}(\%)$ & $\mathrm{n}(\%)$ & $\mathrm{n}(\%)$ & $\mathrm{n}(\%)$ \\
\hline INMP & $251(27,4)$ & $201(80,1)$ & $199(79,3)$ & $116(46,2)$ & $50(19,9)$ & $31(12,3)$ & $43(17,0)$ & $2(0,8)$ \\
\hline H. Sergio Bernales & $95(10,4)$ & $94(98,9)$ & $89(93,7)$ & $94(98,9)$ & $1(1,1)$ & $1(1,1)$ & $1(1,1)$ & $4(4,2)$ \\
\hline H. Hipólito Unanue & $79(8,6)$ & $76(96,2)$ & $73(92,4)$ & $76(96,2)$ & $3(3,8)$ & $2(2,4)$ & $3(3,6)$ & 0 \\
\hline H. Dos de Mayo & $49(5,3)$ & $48(98)$ & $6(12,2)$ & $48(98)$ & $1(2)$ & $0(0,0)$ & $1(2,0)$ & $37(75,5)$ \\
\hline H. San Bartolomé & $96(10,5)$ & $94(97,9)$ & $55(57,3)$ & $93(96,9)$ & $2(2,1)$ & $1(1,0)$ & $1(1,0)$ & $3(3,1)$ \\
\hline H. María Auxiliadora & $90(9,8)$ & $89(98,9)$ & $89(98,9)$ & $89(98,9)$ & $1(1,1)$ & $0(0,0)$ & $0(0,0)$ & 0 \\
\hline H. Daniel A. Carrión & $56(6,1)$ & $39(69,6)$ & $40(71,4)$ & $54(96,4)$ & $2(3,6)$ & $0(0,0)$ & $0(0,0)$ & $14(25)$ \\
\hline H. Rebagliati Martins & $97(10,6)$ & $8(8,2)$ & $8(8,2)$ & $0(0)$ & $89(91,8)$ & $67(65,7)$ & $77(75,5)$ & $0(0)$ \\
\hline H. Sabogal Sologuren & $49(5,3)$ & $49(100)$ & $46(93,9)$ & $49(100)$ & $0(0)$ & $0(0,0)$ & $0(0,0)$ & $2(4,1)$ \\
\hline Hospital de la PNP & $16(1,7)$ & $14(87,5)$ & $14(87,5)$ & $14(87,5)$ & $2(12,5)$ & $0(0,0)$ & $0(0,0)$ & 0 \\
\hline Clínica particular A & $22(2,4)$ & $0(0)$ & $0(0)$ & $0(0)$ & $22(100)$ & $0(0,0)$ & $0(0,0)$ & NA \\
\hline Clínica particular B & $16(1,7)$ & $13(81,3)$ & $13(81,3)$ & $6(37,5)$ & $3(18,8)$ & $3(18,8)$ & $3(18,8)$ & $0(0)$ \\
\hline
\end{tabular}

* Porcentaje respecto al total de la población de estudio $(\mathrm{N}=916)$ y los demás porcentajes son respecto al total de RN del estudio para cada establecimiento de salud.

INMP: Instituto Nacional Materno Perinatal; H: hospital; PNP: Policía Nacional del Perú; RN: recién nacidos; RP: reporte de padres; RSS: reporte de servicios de salud; NA: no aplica.

\section{DISCUSIÓN}

La cobertura de vacunación contra el VHB en RN dentro de las 24 horas de vida en los establecimientos estudiados fue de $85,1 \%$ según el RP y $88,7 \%$ según el RSS, resultados que son mayores a lo señalado como valor basal por el MINSA $(79 \%)^{(7)}$ y otros reportes ${ }^{(5)}$. Sin embargo, aún está alejado de la meta ideal establecida por el MINSA ${ }^{(7)}$ y la meta propuesta por el Grupo Técnico Asesor sobre Enfermedades Prevenibles por Vacunación de la OMS y la Organización Panamericana de la Salud (12); ya que sólo un tercio de establecimientos alcanzaron la cobertura esperable ( $95 \%$ o más). Estos resultados no reflejan lo que sucede en entornos extrahospitalarios y en zonas menos favorecidos; Cabezas et al.(13) encontró en comunidades indígenas de la amazonia del Perú, luego de intervenciones de vacunación, una cobertura de $38,8 \%$ en recién nacidos.

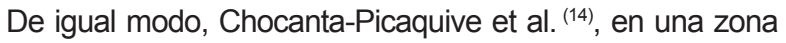
amazónica de Colombia encuentra una cobertura de $30,7 \%$.

Otro hallazgo importante de este estudio, es la identificación de un establecimiento de salud (clínica privada), en la que no se realizaba la vacunación contra el VHB en RN. Este hallazgo es de consideración, dado que existe una normativa nacional de obligatoriedad de la vacunación en instituciones públicas y privadas. La explicación al respecto, puede estar relacionado a la falta de coordinación de dicho establecimiento con las instancias correspondientes del MINSA para la provisión gratuita de las vacunas ${ }^{(7)}$. Otra explicación menos alentadora sería el poco interés en la atención de salud integral materno- perinatal orientada a la prevención de la salud, como es la vacunación contra el VHB en RN.

El presente estudio aporta también valiosa información respecto a la calidad de la atención, lo que se observa principalmente en el Hospital Nacional Dos de Mayo, donde se presenta un alto porcentaje de $\mathrm{RN}$ vacunados dentro de las dos horas de vida, con $100 \%$ de cobertura, según el RSS. En dicho establecimiento se observa un considerable porcentaje de padres que no recibieron información sobre la vacunación de sus hijos $(77 \%)$, lo que hace evidente la necesidad de mejoras en la calidad de la atención, en la provisión de información completa clara y sencilla de la vacunación ${ }^{(7)}$. Por otro lado, se identificó $\mathrm{RN}$ vacunados contra VHB con menos de 2000 gramos, sin tener información de HBsAg materna positiva, que es cuando se recomendaría ${ }^{(8)}$.

Los hallazgos presentados deben ser interpretados tomando en consideración las principales limitaciones del estudio. En primer lugar, el sesgo de información de la hora exacta de la administración de la vacuna contra la infección por VHB. Para minimizar dicho sesgo, la información fue recogida de dos fuentes, de los padres y de los servicios de salud, que fueron mayormente coincidentes. Asimismo, señalar que un porcentaje importante no reporta hora de vacunación, muchos de los cuales, si fueron vacunados, por lo que se observan limitaciones en el registro de la información y en el acceso a la información de los padres. Otra limitación está relacionada a la accesibilidad de la información de las fuentes señaladas, que por la organización jerárquica de 


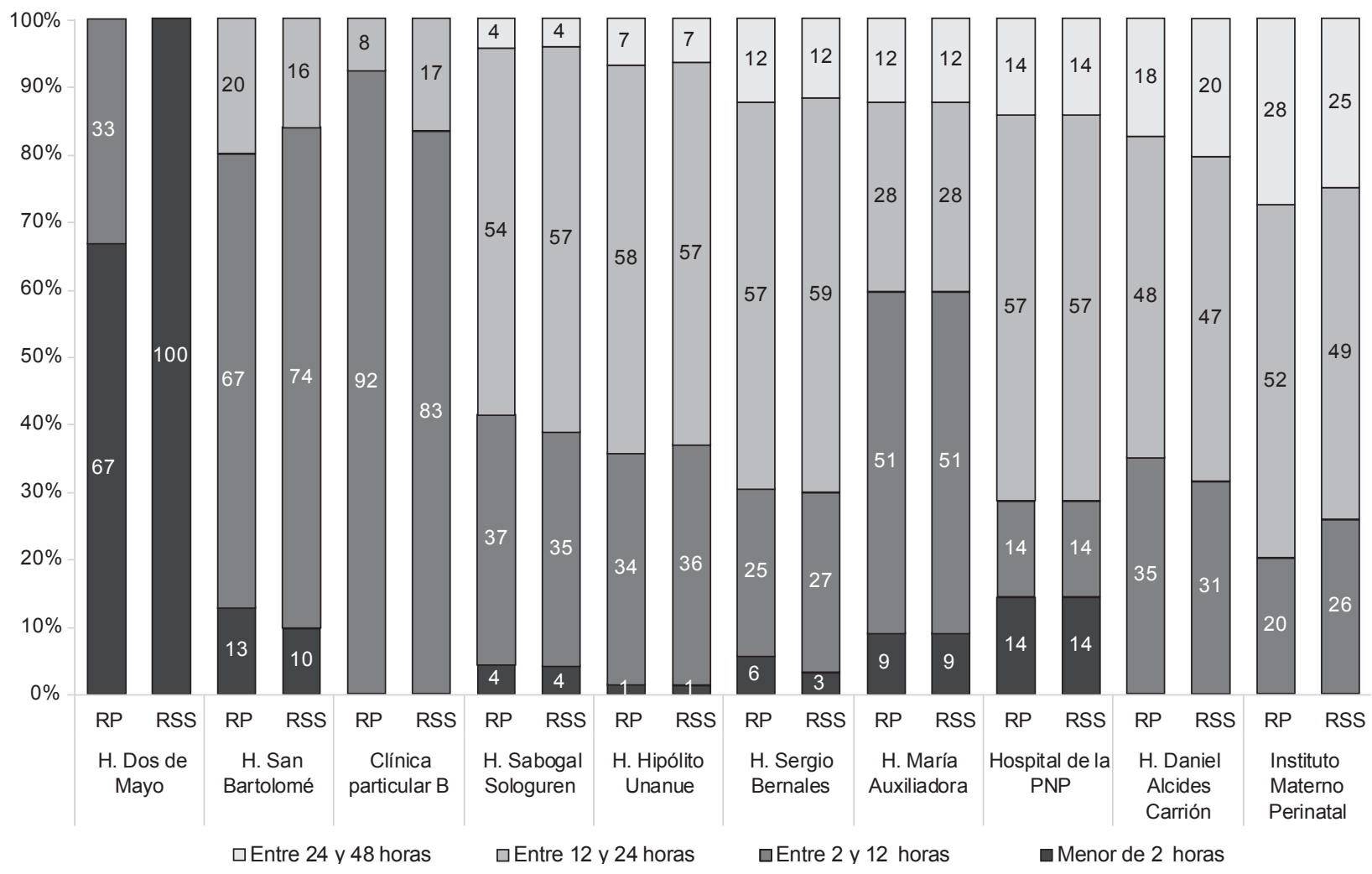

Figura 2. Cumplimiento de vacunación contra el VBH en RN según establecimientos de salud y hora de vacunación ( $n=740)$. RP: reporte de padres; RSS: reporte de servicios de salud; H: hospital; PNP: Policía Nacional del Perú.

No se reportan resultados del H. Rebagliati por la alta proporción de recién nacidos sin reporte de hora de vacunación (91,8\%) y de la Clínica particular $\mathrm{A}$ al no haber realizado ninguna vacunación.

la atención hospitalaria y resguardo de la información, se realizaron coordinaciones con las autoridades y personal, lo que podría haber condicionado cambios en el trabajo habitual del personal de salud.

En conclusión, los resultados muestran una cobertura importante de la vacunación contra el VHB en RN dentro de las 24 horas de vida, aunque aún alejadas de las metas ideales propuestas por el MINSA y la OMS/OPS. En ese sentido, se requiere seguir fomentando la prevención de la trasmisión materno-perinatal del VHB, a través de intervenciones en las gestantes y recién nacidos, lo cual requiere la participación y compromiso de los diversos sectores, incluido el sector privado.
Agradecimientos: los autores agradecen la colaboración de la Dra. Elsa Mantilla Portocarrero, Dr. Víctor Garay Gutiérrez, además de Mariela Yaya Ríos, Verónica Hurtado Vela y Luis Bravo Chauca, en la recolección de la información.

Contribuciones de autoría: $\mathrm{ABO}$ ha participado en la concepción del artículo, la recolección y análisis de datos, redacción y aprobación de la versión final. AFF participo en la concepcion de la investigación, analisis de datos y aprobacion de la version final. EMS, MDA, CBS y FDT han participado recoleccion y análisis de datos, redacción y aprobación de la versión final.

Fuentes de financiamiento: Instituto Nacional de Salud.

Conflicto de intereses: Los autores declaran no tener ningún conflicto de interés.

\section{REFERENCIAS BIBLIOGRÁFICAS}

1. World Health Organization. Hepatitis B [Internet]. Geneva: OMS; 2018 [citado el 6 de marzo de 2018]. Disponible en: http://www.who.int/mediacentre/ factsheets/fs204/es/.

2. World Health Organization. Hepatitis $\mathrm{B}$ vaccines: WHO position paper
- July 2017 [Internet]. Geneva: OMS; 2017 [citado el 6 de marzo de 2018]. Disponible en: http://apps.who.int/ iris/bitstream/handle/10665/255841/ WER9227.pdf; jsessionid=522F830D8513B0A07EDEFB00B40703AA?sequence $=1$.
3. Greenberg DP. Pediatric experience with recombinant hepatitis $B$ vaccines and relevant safety and immunogenicity studies. Pediatr Infect Dis J. 1993;12(5):438-45.

4. Hadler SC, Margolis HS. Hepatitis B immunization: vaccine types, efficacy, 
and indications for immunization. Curr Clin Top Infect Dis. 1992;12:282-308

5. Ropero-Álvarez AM, Pérez-Vilar S, PacisTirso C, et al. Progress in vaccination towards hepatitis B control and elimination in the Region of the Americas. BMC Public Health. 2017;17(1):325. doi: 10.1186/ s12889-017-4227-6.

6. Schillie S. Prevention of Hepatitis B Virus Infection in the United States: Recommendations of the Advisory Committee on Immunization Practices. MMWR Recomm Rep. 2018;67(1):131. doi: 10.15585/mmwr.rr6701a1.

7. Perú, Ministerio de Salud. Resolución Ministerial $\mathrm{N}^{\circ}$ 651-2016/MINSA. Norma Técnica de Salud que establece el Esquema Nacional de Vacunación. Lima: MINSA; 2016.

8. Sociedad Española de Neonatología. Protocolo de seguimiento para el recién nacido menor de $1500 \mathrm{~g}$ o menor de 32 semanas de gestación [Internet]. Madrid: Sociedad Española de Neonatología; 2017 [citado el 9 de septiembre de 2018]. Disponible en: https://www.se-neonatal.es/ Portals/0/Publicaciones/Protocolo-Seguimiento\%20recien\%20nacido_SENeoOK-web.pdf.
9. World Health Organization. Global Health Sector Strategies for HIV, viral hepatitis, STIs, 2016-2021 [Internet]. Geneva: OMS; 2016 [citado el 1 de marzo de 2018]. Disponible en: http://www. who.int/hiv/strategy2016-2021/en/.

10. Ministerio de Salud. Dosis y cobertura de vacunación por edades y tipo de biológico, según departamentos, Perú - Año 2013 [Internet]. Lima: Oficina General de Estadística e Informática, MINSA; 2014 [citado el 1 de marzo de 2018]. Disponible en: http:// www.minsa.gob.pe/estadisticas/estadisticas/Servicios/Inmunizaciones/ VACUMacros.asp?00.

11. Instituto Nacional de Estadística e Informática. Perú: Natalidad, Mortalidad y Nupcialidad, 2015 [Internet]. Lima: INEI; 2016 [citado el 9 de septiembre de 2018]. Disponible en: https://www. inei.gob.pe/media/MenuRecursivo/ publicaciones_digitales/Est/Lib1407/ libro.pdf

12. Technical Advisory Group. Reports of the Technical Advisory Group (TAG) on Vaccine-preventable Diseases [Internet]. Washington, DC: Pan American Health
Organization; 2018 [citado el 8 de marzo de 2018]. Disponible en: http://www. paho.org/hq/index.php?option $=$ com content\&view $=$ article\&id $=1862 \% 3$ Atechnical-advisory-group\&catid $=1549 \% 3$ Ainformation-products\&Itemid=39430\&lang=en.

13. Cabezas-Sánchez C, Trujillo-Villarroel O, Zavaleta-Cortijo C, et al. Prevalence of hepatitis $B$ infection in children under 5 years old on indigenous communities of the Peruvian Amazonia after immunization interventions. Rev Peru Med Exp Salud Publica. 2014;31(2):204-10.

14. Choconta-Piraquive LA, De la Hoz-Restrepo F, Sarmiento-Limas CA. Compliance with birth dose of Hepatitis $B$ vaccine in high endemic and hard to reach areas in the Colombian amazon: results from a vaccination survey. BMC Health Serv Res. 2016;16:293. doi: 10.1186/ s12913-016-1542-z.

Correspondencia: Alfredo Borda-Olivas. Dirección: Avenida Olavegoya 1879, int. 1301. Jesus Maria, Lima 11. Perú. Teléfono: +0511959065633 Email:alfredoborda@gmail.com

\title{
Ahora nuestra revista incluye:
}

\author{
Publicación anticipada \\ Compartiendo publicaciones científicas con el ciudadano \\ $\checkmark$ Videos de presentaciones conjuntas del Instituto Nacional de \\ Salud y la Academia Nacional de Medicina \\ Galería fotográfica y videos
}

https://rpmesp.ins.gob.pe

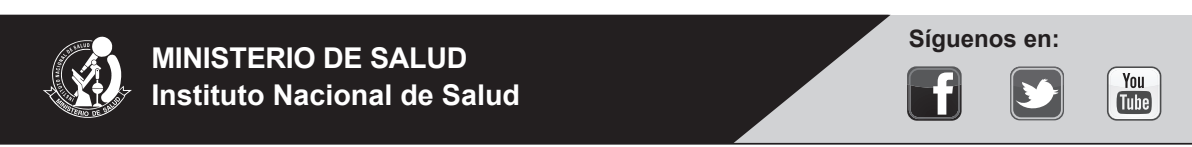

